\title{
BERGAMOT
}

\section{A Green and Multifunctional Asset Exclusive from the Province of Reggio Calabria}

\author{
Francesco Saverio Nesci ${ }^{1, a}$, Natalia Sapone $e^{1, b}$ \\ ${ }^{1}$ Mediterranea University of Reggio Calabria - Department of Agricultural Sciences - \\ Località Feo di Vito - 89124 Reggio Calabria - Italy \\ áfrancesco.nesci@unirc.it \\ bnatalia.sapone@unirc.it
}

Keywords: Bergamot, Multifunction, Associations; Profitability; Land Value.

\begin{abstract}
Bergamot is a typical citrus fruit that comes almost exclusively from the province of Reggio Calabria. It is a symbol of biodiversity and Calabria has a corner on this exclusive asset as more than $90 \%$ of bergamot world production comes from this region . It is to be considered a green asset as it fully complies with Green economy standards and, as every part of the fruit can be used in different sectors, it is a multifunctional product.

Essence is requested by perfumer factories, it is widely employed in the cosmetic sector and in pharmacopoeia. The fresh fruit is gaining growing importance for its therapeutic/health fanatic capacity; the juice is used in pharmacopoeia and in the production of derived products as citric acid, while the peel is used to extract pectin; "pastazzo", which is mainly used as animal feed, is now used in the preparation of a special lime that, according to the oral building tradition handed down in the ionic area, is to be considered important for its antifungal and antibacterial properties, thus in compliance with green economy standards.

Associations have always been in charge of the protection of this product; in the past, they were well represented by the Consorzio del Bergamotto whereas the baton has been recently passed on to UnionBerg O.P.; founded in 2004, it aims at guaranteeing a continuing quality production and fixed prices as required by customers, by means of a "voluntary stockpile".

From an economic point of view, the product profitability is interestingly mirrored in the land value, which is remarkably higher than table values. Sentence 181/2011 of the Constitutional Court declared it unlawful and provides that only the market value is to be considered.

Bergamot is a resource of the coming metropolitan area and it is therefore worth the attention of scientific research as it is likely to offer further and determinant contributions in less explored sectors, especially those pertaining to health and wealth.
\end{abstract}

\section{Presentation}

Bergamot, the origins of which appear extremely uncertain, is a typical citrus fruit that comes almost exclusively from the province of Reggio Calabria. It is a symbol of biodiversity and it represents ancient territories and places where quality agriculture, restoration projects aimed at ancient villages, sustainable tourism and traditional cultures blend together in harmony.

Calabria has a corner on this exclusive asset as more than $90 \%$ of bergamot world production comes from this region; the remaining $10 \%$, due to specific climate requirements, comes from some specific African (Costa d'Avorio, Malì, Camerun, Guinea) and South American regions (Argentina and Brazil) but they never meet the high Calabrian standards in terms of fragrance.

It is to be considered a green asset as it fully complies with Green economy standards like "creating wealthier conditions and social equity, without putting the environment at risk nor exploiting the decreasing poor ecologic sources, reducing carbon, being efficient in the detection of sources and being socially inclusive". On the one hand, it facilitates the increase of regional GDP levels, occupying the first positions in the ranking of exported chemical products; on the other hand, 
it doesn't damage the environment as every part of the fruit can be used in different sectors, making it a multifunctional product.

Farming currently spreads over an area of about 1.000/1.200 ha developing along the narrow coastline, enclosed between the Aspromonte slopes and the sea; as this area has been increasingly featured by urbanization, farming insinuated in the several torrent valleys of this land, ranging from the village of Villa San Giovanni, on the Tyrrhenian Sea, to the border of the Province of Catanzaro, on the Ionian sea [1].

Official Istat data define a farming surface to be about 1.500 ha in 2010 whereas, according to the information provided by the sector representatives, $1.100-1.200$ ha would be a reliable estimate of the bergamot farming area (INEA). Farms are mainly traditional companies, featured by low level processes and scarce technology contribution.

\section{History of colture}

In the early $1920 \mathrm{~s}$, farming spread over an area of about 3.300 ha and it widened to reach 3.500 ha in the 1960s; it has since been reduced to the current 1.000 ha due to market vicissitudes, structural weaknesses of local companies, compact demand, smear campaigns causing recurring crisis that, from the 1970s onwards, has generated a growing disaffection toward farming and an inclination towards a disorganized urban development [2].

This meaningful reduction of farming areas has caused a consequent reduction of a $1 / 3$ of the produced essence that currently equals 100.t./year. On the contrary, about $3.000 \mathrm{t}$. /year are sold worldwide to be mixed with poor quality and low cost essential oils or with synthetic chemical products.

Associations have always been in charge of the protection of this product; in the past, they were well represented by the Consorzio del Bergamotto whereas the baton has been recently passed on to UnionBerg O.P.; founded in 2004, it aims at guaranteeing a continuing quality production and fixed prices as required by customers, by means of a "voluntary stockpile". The effectiveness of this policy was confirmed by the analysis of sale volumes as they appear to have increased from $€$ 401.000 in 2008 to 3 million euros in 2012. It can be interpreted as a sign of growing trust among producers [3].

In 2007, Unionberg O.P. itself established the "Consorzio di Tutela del Bergamotto di Reggio Calabria"; this means that, among its duties, it will manage and protect the exploitation of DOP as provided by L. 39/2000 and confirmed by EU Reg. 509/01, in order to guarantee more effective protection of the quality of the product.

At the same time, this policy generated a stabilization of costs and salaries for all the actors involved in the production chain. It amounts to $€ 40$ /q. of fruit and to more than $€ 1,50$ in the case of organic and DOP products within the ongoing 2013/2014 campaign.

With reference to prices, analyzing official data released by the Consorzio between 1951 and 1990 and comparing it to data compiled by companies and producers, it is interesting to note their gradual increase in terms of current prices while fixed prices in euro (2012) appear to report a continuing recession. Essence value in the $1950 \mathrm{~s}$ was $6,60 € / \mathrm{kg}$ in current prices whereas in fixed prices (2012) it amounts to about $205 € / \mathrm{kg}$. In fact, despite euro loosing buying power of about 30 points between 1951 and 2012, the current quotation of the essence, equal to $80 / 83 € / \mathrm{kg}$, has been reduced to more than $60 \%$ compared to the value it held at the beginning of the mentioned period, if considered in terms of fixed prices.

\section{The multifunctional role of the culture}

In this regard, it seems appropriate to highlight how we usually refer to the value of the fruit/essence, without taking into any account the potential added value of all the other characteristics of this product which make it ascribable as multifunctional according to green economy standards. 
Essence is the main product and it is squeezed out of the opercula located inside the epicarp of the fruit. Requested by perfumer factories, it is widely employed in the cosmetic sector to produce deodorants, anti-dandruff lotions, suntan creams, hygiene products, in pharmacopoeia for its antiseptic, antibacterial, diuretic and dermatological characteristics, for its power to reactivate blood flow and in the production of hygiene antiviral products.

The consumption of the fresh fruit is gaining growing importance for its therapeutic/health fanatic capacity to cut down cholesterol levels. The fresh fruit price in the 2013/14 campaign varied between 1,5 and $2 € / \mathrm{kg}$, being definitely higher than the price set by industries. New products have been developed also in confectionary and liquor sectors for the unique aroma that bergamot provides and for the same reason it is consistently used in gastronomy.

Byproducts are also widely employed in different fields; in the case of the juice, it is used in pharmacopoeia and in the production of derived products as citric acid for non-alcoholic drinks, food and dye; the peel is used to extract pectin, employed as a gelatin maker or in pharmaceutics (hemostatic products, antidiarrheal, etc. ); "pastazzo", which is mainly used as animal feed, is made of dried and processed waste. Thanks to the research conducted by the University of Reggio Calabria, "pastazzo" is now used in the preparation of a special lime that, according to the oral building tradition handed down in the ionic area, is to be considered important for its antifungal and antibacterial properties, thus in compliance with green economy standards.

\section{The value of land}

From an economic point of view, the product profitability is interestingly mirrored in the land value. It currently varies from a minimum of $50.000 € /$ ha to a maximum of $80.000 € /$ ha according to the agronomic conditions of the station and its value, within a real estate business that is poorly active.

Using analytic assessment tools based on the capitalization of future income, the resulting value would reach the highest levels and, as a fact, the selected discount rate would become determinant when calculating the final result. Defining the net flow, as resulting from the sum of income and expenses incurring over 50 years of activity for an average company in ordinary conditions, the fund value, determined capitalizing periodic income with a rate of $2.0 \%$, as applied in agriculture, would equal $78.000 € /$ ha and it is destined to decrease when coefficients are higher. As a matter of fact, when determining the selling price of bergamot, the cost of the fruit intended for industry is taken into account and not the possible increase due to its selling for fresh consumption nor the other rises deriving from an appropriate valorization of byproducts $[4,5]$.

With reference to VAM (farming average values) as set by the Commissione Provinciale Espropri, as they represent the standard for compensation in case of expropriation of farming areas, bergamot farming areas in r.a. 8 (metropolitan area of Reggio Calabria) were worth $75.000 € /$ ha (13\% higher than average citrus farming) in 2012. In this regard, it is interesting to highlight the speculation on the value of the same farming if located in bordering areas, although a significant difference in terms of agronomic value is not detectable. Such variability of more than $50 \%$ can be explained only in terms of "revenue of a position".

The benefits system regulated by art. 15 and 16 L. 865/71, then provisions 1 and 3 of art. 40 of DPR. n. 327 - 8 June 2001 "Testo unico", in place since 2003, provided that "average farming value" was to be taken into account when defining the amount of temporary compensation and that the real farming value was to be taken into account when defining the amount of final compensation.

Sentence 181/2011 of the Constitutional Court declared provisions 2 and 3 of art. 40 D.P.R. 327/2001 (T.U.), before art. 15 and 16 of L. 865/71., unlawful, pointing out that in order to determine the amount of compensation for expropriation only the market value is to be considered, without taking into account the characteristics of the expropriated product (whether for construction or not).

This sentence represents a novelty in the field of benefits for non-building areas that still keep a potential extra-farming usage, as it states that «although it can't be denied that market value and 
farming average value sometimes correspond, the former value is often (and sometimes remarkably) higher than the latter, as land attractiveness on the market not only depends on it being a building area, but also on many other factors, especially its position and its concrete possibilities to be exploited for purposes other than farming ».

\section{Conclusions}

With reference to the observations made on the economic value of the fruit, especially concerning its being a multifunctional asset, it stands out that bergamot is an important resource of the coming metropolitan area and it is therefore worth the attention of scientific research as it is likely to offer further and determinant contributions in less explored sectors, especially those pertaining to health and wealth.

The establishment of a Higher International Institute for Perfumery, Cosmetics and Spices, funded L. 246/89, will provide further support to the product valorization, also in terms of image, even if it is difficult to hypothesize a strategy to catch up to 5 centuries of the French School of fragrance and cosmetics.

The power of associations represents the keystone of this product and in order to protect it, the following commitments are necessary: time, strategic consistency, knowledge of market regulations, involvement and ethics of all the actors, also by complying with the regulations set as this is the only way to achieve positive results.

\section{References}

[1] P. Amato: Storia del bergamotto di Reggio Calabria - Ed. Città del Sole, (2005)

[2] F.S. Nesci, N. Sapone, M. Baldari: Tutela e sviluppo del bergamotto reggino - Atti della XXXII Conferenza scientifica annuale dell'Associazione Italiana di Scienze Regionali Torino, (2011)

[3] F.S. Nesci: Il bergamotto: dal'azienda agraria al mercato. Problemi attuali e prospettive future - Estratto da Essenze e Derivati Agrumari - n.3 - 2001

[4] V. Gallerani, G. Zanni, D. Viaggi: Manuale di Estimo, Ed. McGraw-Hill, Milano, (2004)

[5] I. Michieli, M. Michieli: Trattato di Estimo - Ed agricole, Bologna, (2002) 